\title{
Retracted: Optimal Coordinated Energy Management in Active Distribution System with Battery Energy Storage and Price-Responsive Demand
}

\author{
Mathematical Problems in Engineering
}

Received 9 December 2022; Accepted 9 December 2022; Published 4 January 2023

Copyright ( 2023 Mathematical Problems in Engineering. This is an open access article distributed under the Creative Commons Attribution License, which permits unrestricted use, distribution, and reproduction in any medium, provided the original work is properly cited.

\begin{abstract}
Mathematical Problems in Engineering has retracted the article titled "Optimal Coordinated Energy Management in Active Distribution System with Battery Energy Storage and Price-Responsive Demand" [1]. The journal was contacted by an author group who stated that this article is a duplication of a submitted but unpublished manuscript, published without their permission. This group provided the appropriate funding documentation and underlying data in support of their claim, while the authors listed on the article were unresponsive to our requests for clarifications. The article is therefore being retracted from the journal due to these concerns with the agreement of the Editorial Board.
\end{abstract}

\section{References}

[1] Y. Xing, H. Zhao, Z. Shen et al., "Optimal Coordinated Energy Management in Active Distribution System with Battery Energy Storage and Price-Responsive Demand," Mathematical Problems in Engineering, vol. 2021, Article ID 6620550, 12 pages, 2021. 


\title{
Optimal Coordinated Energy Management in Active Distribution System with Battery Energy Storage and Price-Responsive Demand
}

\author{
Yahong Xing $\mathbb{D}^{1},{ }^{1}$ Haibo Zhao $\mathbb{D}^{1},{ }^{1}$ Zeyuan Shen $\mathbb{D D}^{1}{ }^{1}$ Lin Zhang ${ }^{\mathbb{D}},{ }^{1}$ Zhi Zhang $\mathbb{D}^{1}{ }^{1}$ Qi Li $\mathbb{D}^{1},{ }^{1}$ \\ and Se $\mathrm{Wu}$ (D) $^{2}$ \\ ${ }^{1}$ Economic and Electrical Research Institute of Shanxi Electrical Power Company of SGCC, Taiyuan 030002, China \\ ${ }^{2}$ Shanxi Electrical Power Company of SGCC, Taiyuan 030001, China
}

Correspondence should be addressed to Lin Zhang; zhanglin20201024@126.com

Received 28 October 2020; Revised 15 December 2020; Accepted 6 February 2021; Published 22 February 2021

Academic Editor: Zhixiang Zou

Copyright $\odot 2021$ Yahong Xing et al. This is an open access article distributed under the Creative Commons Attribution License, which permits unrestricted use, distribution, and reproduction in any medium, provided the original work is properly cited.

Contemporary distribution networks can be seen with diverse dispatchable and nondispatchable energy resources. The coordinated scheduling of these dispatchable resources, together with nondispatchable resources, can provide several technoeconomic and social benefits. Since battery energy storage systems (BESSs) and microturbine units (MT units) are capital-intensive, a thorough investigation of their coordinated scheduling under the economic criterion will be a challenging task while considering dynamic electricity prices and uncertainties of renewable power generation and load demand. This paper proposes a comprehensive methodological framework for optimal coordinated scheduling of BESSs with MT unit considering existing renewable energy resources and dynamic electricity prices to maximize the daily profit function of the utility by employing a recently explored modified African buffalo optimization algorithm. The key attributes of the proposed methodology are comprised of mean price-based adaptive scheduling embedded within a decision mechanism system (DMS) to maximize arbitrage benefits. DMS keeps track of system states as a priori, thus resulting in an artificial intelligence-based solution technique for sequential optimization. Further, a novel concept of fictitious charges is also proposed to restrict the counterproductive operational management of BESSs. The proposed model and method are demonstrated on the 33-bus distribution system, and the obtained results verify the effectiveness of the proposed methodology.

\section{Introduction}

Modern distribution systems can be seen with high penetration of distributed generations (DGs) on account of several technoeconomic and social concerns. These DG units may exist in a combination of nondispatchable sources, i.e., solar photovoltaics (PVs) and wind turbines (WTs), and dispatchable sources such as MT units and BEESs. The coordinate scheduling of deployed BESSs and MT units in the uncertain renewable energy source (RES) batteries and motor system can shift the peak load, improve the absorption level of intermittent renewable energy, enhance the reliability of power system, delay the investment of power equipment, and so on $[1,2]$. However, the escalating presence of BESSs requires scheduling and optimizing their coordination and management $[3,4]$. This is because such coordinated management can absorb intermittency of renewable power generation besides significant arbitrage benefits under dynamic electricity pricing owing to their flexibility of dispatching energy at an appropriate time. Since BESSs are capital-intensive, their coordinated management must involve the optimization of the daily profit function of the utility. Therefore, arbitrage benefits and the desired operating strategy of dispatchable sources are the key issues that must be taken care of while solving the decision problem for profit maximization. Nevertheless, the problem is challenging on account of the dynamic behaviour of the generation from RESs, demand, and electricity market that led to further added computational process of BESSs which needs adaptive technique to handle [5]. The consideration of dynamic electricity price may further add computational burden to the optimization technique on account of dynamically varying system states with a large number of state variables [6]. 
A large number of research studies have been done to investigate the coordinated management of energy storage devices with diverse DERs using different charging/discharging strategies and artificial intelligence- (AI-) based optimization techniques. In most of the literature studies, the scheduling of storage technologies is governed either by the objective function to be optimized or sometimes by time-dependent fixed operating strategies. Several works [7-13] have been reported for coordinated management of energy storage devices with diverse DERs using different charging/discharging strategies and AI-based optimization techniques. In most of the literature studies, the scheduling of storage technologies is governed either by the objective function to be optimized or sometimes by time-dependent fixed operating strategies. In $[6,14,15]$, dynamic programming is employed for optimal scheduling of BESSs under uncertain environment. The problem is evaluated for minimization of a cost function to ensure flexible and economical utilization of BESSs. The authors concluded that practically large-scale BESS utilization could be achieved while optimizing consumption from RESs. The optimal utilization of BESSs involves charging/discharging intervals in accordance with dynamic demand, renewable power generation, and pricing signal. In most of the abovementioned works, these intervals are kept fixed on the basis of the aggregated demand curve. Such battery operation strategy may lead to low profit and increased energy losses since the profiles of renewable penetration, demand, and electricity price vary on a daily basis [16]. However, a flexible and adaptive operational strategy for BESSs together with uncertainty modelling of load demand and RES generation may provide better utilization of existing energy resources and enhanced arbitrage benefits. The scheduling of BESS in dynamic electricity pricing environment, therefore, essentially considers arbitrage benefits, which have not been fully exploited, in order to justify their installation. The arbitrage benefits have been investigated by limited researchers [17-20]. From the aforesaid discussion, it can be summarized that the scheduling problem of BESSs with MT unit must be coordinated with the existing RESs considering dynamic pricing signal. However, when the BESSs are utility-owned, the daily profit function has not been taken into consideration. The profit function is discontinuous and nonlinear and encompasses several system states together. Therefore, it cannot be efficiently optimized with less computational burden using an AI-based natureinspired metaheuristic technique, like GA or PSO, unless it is supplemented by suitable means to restrict enormous problem search space.

This paper proposes a modified methodology for optimal coordinated management of utility-owned BESSs with MT unit under dynamic electricity pricing environment to maximize the daily profit function (DPF) of the utility while fully exploiting existing DERs in the distribution system. The DPF is maximized using a recently explored swarm intelligence-based optimization technique, namely, modified African buffalo optimization (MABO) with high investigation and utilization competencies. The salient contributions of the proposed methodology are the mean price-based adaptive scheduling (MPAS) of BESSs, decision mechanism system (DMS), and fictitious charges (FCs) while considering uncertainties in renewable power generation and load demand. MPAS provides price-based dynamic charging and discharging intervals for BESSs. DMS tracks all the system states to gather and process the information as a priori and thus guides $\mathrm{MABO}$ for sequential optimization. This may reduce the computational burden and may also enhance the efficacy of the AI-based solution technique. In addition, the methodology employs a novel concept of fictitious charges (FCs) to check the uneconomic operational management of BESSs. All these features facilitate desired operation of utility-owned BESSs while optimizing profit function. From the current research, these characteristics have not been studied yet. The investigation and comparison of the application results on a stander 33-bus test distribution system verify the effectiveness of the proposed methodology.

\section{Mean Price-Based Adaptive Scheduling}

Modern-day distribution systems are having integrated dispatchable and nondispatchable distributed energy resources, which may include SPVs, WTs, BESSs, and MT units. The integration of diverse DER technologies is vital for the optimal operation of current distribution systems. However, the proper coordination among multiple DERs is essential from both the operational and economic efficiency point of view of the current distribution systems. For better economic efficiency, RESs must be fully tapped and utilized locally $[18,21]$. In this context, the optimal management of energy storage system (ESS) has been acknowledged as an amicable solution. BESSs are getting more and more attention and application in distribution system because of its characteristics of bidirectional charging [19-24]. This ability is crucial while considering dynamic electricity price as BESSs can store energy during off-peak hours and deliver the same during peak hours. However, if MT unit is used in coordination with BESSs, better results may be achieved while considering forecasting errors in load demand and power generation from RESs [18]. With these concerns, BESSs and MT unit can be optimally scheduled to maximize the technoeconomic efficiency of the distribution systems while maintaining several operational constraints.

Considering the actual situation of the electricity market, this paper assumes that PVs and WTs are owned by a third party such as DGOs, while MT unit and BESSs are owned by the utility. The utility purchases electricity from the grid with day-ahead dynamic electricity price and from DGOs with the contract price. The utility sells electricity to consumers at the dynamic day-ahead price, earning extra revenue while guaranteeing a definitely fixed margin of profit. When MT unit is added to the power system, it can supply power to the grid during peak price periods and store electricity during low price periods for profit.

However, considering the intermittency of RESs, stochastic nature of the load demand, charging/discharging status of BESS, forecasting errors in load demand and renewable power generation, and other factors, BESS with MT 
optimization scheduling implementation is a very complex problem. These issues essentially require the consideration of dynamically varying system states which may be very large in number over the time frame of the problem, say over a sample day. In order to maximize the DPF, RESs as well as dynamic electricity pricing signal should be comprehensively considered for optimal scheduling. This imposes real challenges owing to the optimal utilization of existing DERs. Such complex nonlinear optimization problems can be effectively handled using AI-based nature-inspired metaheuristic techniques but are highly computationally demanding due to simultaneous optimization of a large number of system states. Otherwise, accuracy may suffer. Alternatively, sequential optimization may be adopted. However, sequential optimization evolves difficulties because the AI-based solution technique is optimizing the objective function for the current state while the rest of the states remained unsighted. It may eventually result in loss of coordination control over the charging/discharging status of BESS while considering dynamic electricity pricing. It happened because the status of BESS will be decided by the profit function of that particular state without considering the profit function of the day as the BESS is utility-owned. This method has great possibilities to cause underutilization of BESS or alleviate battery lifetime, and it is highly undesirable. Therefore, necessary constraints should be added to the sequential optimization method to look after all system states.

2.1. Decision Mechanism System. The price tariff is chosen to be the decision vector in our work to evaluate the scheduling of DERs to maximize profit function because of its direct, flexible, and attractive nature. A DMS is developed that suggests a suitable heuristic-based charging/discharging strategy of BESS over the scheduling period, as a priori, while considering a dynamic electricity price. In this mechanism, the mean price plays as the decision vector in deciding the charging/discharging status of BESS and thus helps to avail price and energy arbitrage benefits. With this price decision vector, the possible power transactions from controlled DERs now can be assessed easily throughout the scheduled period. In this way, DMS guides the AI-based solution technique for other system states. For instance, the decision about the charging and discharging mode of BESSs may be governed by the following respective equations:

$$
\begin{array}{ll}
E_{G}(\Delta t i)<\overline{E_{G}} ; \quad \forall \Delta t i \in T_{\mathrm{BESS}}^{C}, \\
E_{G}(\Delta t i)>\overline{E_{G}} ; \quad \forall \Delta t i \in T_{\mathrm{BESS}}^{D},
\end{array}
$$

where $E_{G}(\Delta t i)$ denote the electricity price in the distribution system; $\overline{E_{G}}$ denote the mean price, and $T_{\mathrm{BESS}}^{C}$ and $T_{\mathrm{BESS}}^{D}$ denote the charging and discharging time of BESS, respectively.

2.2. BESS Management. The abovementioned equations merely decide the charging or discharging status of BESS. For economical operation, the scheduling of BESS over the subperiods $T_{C}^{\mathrm{BESS}}$ and $T_{D}^{\mathrm{BESS}}$ should consider dynamic price to enhance DPF. This needs identification of those particular states where either charging or discharging of BESS is optimum. Furthermore, there is a need to address boundary conditions of BESS dispatches for these selected states so that it can be supplied whenever demanded by the solution technique. Therefore, the following simplified algorithm is proposed in this paper:

(1) The sequence of discharging states is obtained by arranging all the system states above the mean price in descending order.

(2) Subsequently assign dispatch limit $P_{\mathrm{BESS}, b}^{\max , D}$ for $b$ th BESS, on priority, for these discharging states till SOC exhausted. However, the dispatch allocation for the final discharging state being selected may not be $P_{\mathrm{BESS}, b}^{\max , D}$, which can be evaluated as

$$
\begin{aligned}
& \qquad \frac{\left(\mathrm{SOC}_{b}^{\max }-\mathrm{SOC}_{b}^{\min }\right) W_{\mathrm{BESS}, b}^{R}-\gamma P_{\mathrm{BESS}, b}^{\max , D}}{\eta D}, \\
& \text { where } \\
& \gamma=\text { floor }\left(\frac{\left(\mathrm{SOC}_{b}^{\max }-\mathrm{SOC}_{b}^{\min }\right) W_{\mathrm{BES}, b}^{R}}{P_{\mathrm{BESS}, b}^{\max , D}}\right) .
\end{aligned}
$$

(3) The dispatch limit for the remaining discharging states is set to zero.

While considering decisions about the charging of BESS, the same algorithm can be modified as described below:

(1) The sequence of charging states is obtained by arranging all system states below the mean price in ascending order.

(2) Subsequently assign dispatch limit of charging

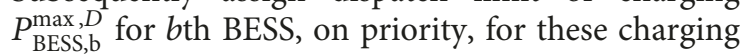
states till the upper limit of SOC reached. However, the dispatch allocation for the final charging state being selected may not be $P_{\mathrm{BESS}, b}^{\max , D}$, which can be evaluated as

$$
\frac{\left(\mathrm{SOC}_{b}^{\max }-\mathrm{SOC}_{b}^{\min }\right) W_{\mathrm{BESS}, b}^{R}-\xi P_{\mathrm{BESS}, b}^{\max , C}}{\eta_{C}}
$$

where

$$
\xi=\text { floor }\left(\frac{\left(\mathrm{SOC}_{b}^{\max }-\mathrm{SOC}_{b}^{\min }\right) W_{\mathrm{BESS}, b}^{R}}{P_{\mathrm{BES}, b}^{\max , \mathrm{C}}}\right) .
$$

(3) The charging limit for the remaining states is set to zero.

In this way, DMS helps the optimization techniques to ensure full discharging/charging of BESS during the highest price band/lowest price band of the day. System states where no power transaction of BESSs takes place will be representing the standby mode. Such standby mode between the consecutive charging and discharging modes of BESS is beneficial. It helps to alleviate the inconvenience caused to 
the operation of converters interconnected to the BESSs due to direct switching from charging mode to discharging mode and vice versa [15].

2.3. Fictitious Charges (FCs). Since the BESS is assumed to be utility-owned, practically no revenue is received by the utility against BESS charging, and no charges shall be paid by the utility while it delivers energy into the system. With this concern, BESS would not charge at all because then actual charges have to be paid by the utility to either grid or DGOs and that reduces the profit function of that state. Moreover, BESS will be ready every time to release energy into the system as this reduces the charges to be paid by the utility against energy purchase from the grid or DGOs and thus increases the profit function of that state. This certainly hampers the BESS management process. These difficulties have been overcome in the proposed methodology by introducing a novel concept of fictitious charges (FCs). In fact, FCs will provide a watershed in the optimization process while scheduling the BESSs for profit function maximization. FCs are suggested to impose on BESSs whenever transacting energy with the distribution system. With this ideology, the utility virtually receives revenue FC against each unit charging of BESS. Similarly, the utility shall virtually pay FC for each unit of energy being discharged from the BESS. The FC, $E_{\mathrm{BESS}}^{F}$, is kept fixed at the mean price with the consideration of (1) and (2). The action of introducing FCs avoids unwanted BESS scheduling, as explained as follows. When $E_{G}(\Delta t i)<E_{\mathrm{BESS}}^{F} ; \forall \Delta t i \in T_{\mathrm{BESS}}^{C}$, it facilitates the utility to receive more energy from BESS units against the energy supplied for their charging than that it pays to purchase the same if charging BESS and restrains as the utility receives less revenue from the customers than what it has to pay to BESSs for supplying this energy into the system if discharging. When $E_{G}(\Delta t i)>E_{\mathrm{BESS}}^{F} ; \forall \Delta t i \in T_{\mathrm{BESS}}^{D}$, the situation is reversed. FCs are imposed simply to prevent undesirable operation of BESS during optimization; however, these are deducted before evaluating the fitness of the objective function.
2.4. Operational Strategy for MT. The primary purpose of integrating MT units into a distribution system is to absorb the power imbalance between contract demand and local power generation due to the power generation prediction errors from RESs and load demand [22]. Moreover, sufficient reserve should be kept to deal with forecasting errors, and economic efficiency should also be considered. For the given decisions, the possible dispatches and corresponding dispatch period for MT scheduling while optimizing DPF can be determined using the algorithm suggested in Section 2.2. This information is also transferred to DMS to guide the AI-based solution technique.

\section{Problem Formulation}

3.1. Renewable Generation and Load Demand Modelling. As discussed, the intermittency and variability in renewable power generation and load demand impose particular challenges on the performance of distribution networks. Therefore, in such a volatile environment, for significant improvement in operating performance of DER integrated distribution networks and to achieve more realistic results, an effective way of handling and modelling uncertain data becomes a primary concern for the network operator. Zeng et al. [23] introduced a simple deterministic approach (polyhedral uncertainty sets) to tackle the intermittency of RES generation and load consumption more competently than the conventional stochastic approaches. In [24], the synthetic data are generated from available annual data by utilizing data spread (DS) and a budget of uncertainty (BOU). The DS and BOU are made dynamic, unlike [23] where they are taken as fixed while generating uncertainty sets. These are constructed by using hourly mean and standard deviation (SD) of the monthly data. Further, the modified modelling approach considers in hand the availability of annual data for solar and wind power generation and load demand $[25,26]$.

The mathematical expression of the modified uncertainty set $U_{y, m}^{g / d}(t)$ for renewable power generation and load demand can be defined as

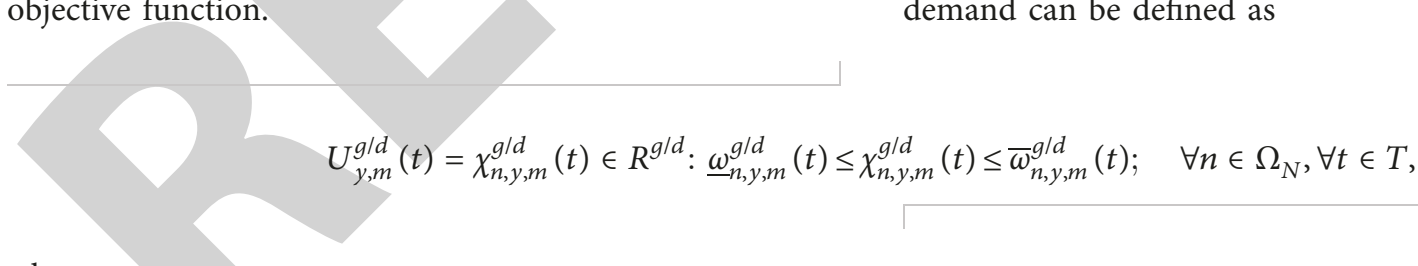

where

$$
\underline{\omega}_{n, y, m}^{g / d}(t)=\omega_{n, y, m}^{g / d}(t)-k \sigma_{n, y, m}^{g / d}(t), \bar{\omega}_{n, y, m}^{g / d}(t)=\omega_{n, y, m}^{g / d}(t)+k \sigma_{n, y, m}^{g / d}(t)
$$

such that

$$
\underline{\mu}_{n, y, m}^{g / d}(t) \leq \widehat{\chi}_{n, y, m}^{g / d}(t) \leq \bar{\mu}_{n, y, m}^{g / d}(t) ; \underline{\mu}_{n, y, m}^{g / d}(t)=\mu_{n, y, m}^{g / d}(t)-k \widehat{\sigma}_{n, y, m}^{g / d}(t), \bar{\mu}_{n, y, m}^{g / d}(t)=\mu_{n, y, m}^{g / d}(t)+k \widehat{\sigma}_{n, y, m}^{g / d}(t) .
$$


In (7)-(9), the available annual data and data to be synthesized are represented by $\omega$-terms and $\chi$-terms, respectively. The DS and BOU are described by the intervals $\left[\underline{\omega}_{n, y, m}^{g / m}(t), \bar{\omega}_{n, y, m}^{g / m}(t)\right]$ and $\left[\underline{\mu}_{n, y, m}^{g / d}(t), \bar{\mu}_{n, y, m}^{g / d}(t)\right]$ at node $n$ during $i$ th system state for $m$ th month of the year as shown in (8) and (9), respectively. Further, the value of $k$ (userdefined coefficient) is taken as unity, as in [24]. The uncertainty sets for PV and WT power generations and load demand are generated from their corresponding available data. For further details about the necessary steps involved in generating uncertainty sets, refs. [23, 24, 27] may be referred.

3.2. Optimal Coordinated Energy Management Modelling. The objective of the proposed function is to maximize the profit for optimal scheduling of BESSs and MT unit while considering $i$ th system state, which is formulated as

$$
\operatorname{Max} O F(t)=R(t)-P(t)-F C(t),
$$

where

$$
\begin{aligned}
R(t)= & P_{D}(t) E_{C}(t) t+P_{\mathrm{loss}}(t) E_{G}(t) t+E_{\mathrm{BESS}}^{F} \sum_{b=1}^{N_{B}} P_{\mathrm{BESS}, b}^{C}(t) t, \\
P(t)= & P_{G}(t) E_{G}(t) t+E_{S P V} \sum_{p=1}^{N_{S P V}} P_{S P V, p}(t) t+E_{W T} \sum_{q=1}^{N_{W T}} P_{W T, q}(t) t+E_{M T} \sum_{r=1}^{N_{M T}} P_{W T, r}(t) t \\
& +M_{M T} \sum_{r=1}^{N_{M T}} P_{M T, r}(t) t+M_{\mathrm{BESS}} \sum_{b=1}^{N_{B}}\left(P_{\mathrm{BESS}, b}^{C}(t)+P_{\mathrm{BESS}, b}^{D}(t)\right) t+E_{\mathrm{BESS}}^{F} \sum_{b=1}^{N_{B}} P_{\mathrm{BESS}, b}^{D}(t) t, \\
F C(t)= & E_{\mathrm{BESS}}^{F} \sum_{b=1}^{N_{B}} P_{\mathrm{BESS}, b}^{C}(t) t-E_{\mathrm{BESS}}^{F} \sum_{b=1}^{N_{B}} P_{\mathrm{BESS}, b}^{D}(t) t .
\end{aligned}
$$

Equation (11) denotes the revenue of utility against the sale of energy, (12) shows the payments by the utility against purchasing power from the grid and DGOs, and (13) represents FCs imposed on BESS energy transactions during the $i$ th state. The objective function defined by (10) is optimized for each state, and then, the DPF is evaluated as follows:

$$
O F_{1}=\sum_{i=1}^{N_{S}} O F(t),
$$

subjected to the following equality and inequality constraints:

(1) Nodal power balance:

$$
\begin{array}{r}
P_{G}\left(\Delta t_{i}\right)+\sum_{p=1}^{N_{S P V}} P_{S P V, p}\left(\Delta t_{i}\right)+\sum_{q=1}^{N_{W T}} P_{W T, q}\left(\Delta t_{i}\right)+\sum_{r=1}^{N_{M T}} P_{M T, r}\left(\Delta t_{i}\right) \mp \sum_{b=1}^{N_{B}} P_{\mathrm{BESS}, b}^{C / D}\left(\Delta t_{i}\right)-P_{D}\left(\Delta t_{i}\right)-P_{\text {loss }}\left(\Delta t_{i}\right)=0 ; \\
\forall \Delta t_{i} \in T_{\mathrm{BESS}}^{C / D}, \forall p \in \Omega_{S P V}, \forall q \in \Omega_{W T}, \forall r \in \Omega_{M T}, \forall b \in \Omega_{B},
\end{array}
$$

where "-" and "+" signs will be used when a BESS is charging and discharging, respectively.

(2) Nodal voltage limits:

$$
\left|V^{\min }\right| \leq\left|V_{k}\left(\Delta t_{i}\right)\right| \leq\left|V^{\max }\right| ; \quad \forall \Delta t_{i} \in T, \forall k \in \Omega_{N} .
$$

(3) Feeders current limits:

$$
I_{j}\left(\Delta t_{i}\right) \leq I_{j}^{\max } ; \quad \forall \Delta t_{i} \in T, \forall j \in \Omega_{E} .
$$

(4) Power dispatch constraints of MT:

$0 \leq P_{M T, r}\left(\Delta t_{i}\right) \leq P_{M T, r}^{R}-P_{M T, r}^{\mathrm{res}} ; \quad \forall \Delta t_{i} \in T_{M T}^{D}, \forall r \in \Omega_{M T}$.

(5) BESS charging constraint:

$0 \leq P_{\mathrm{BESS}, b}^{C}\left(\Delta t_{i}\right) \leq P_{\mathrm{BESS}, b}^{\max , C}\left(\Delta t_{i}\right) ; \quad \forall \Delta t_{i} \in T_{\mathrm{BESS}}^{C}, \forall b \in \Omega_{B}$.

(6) Calculation of maximum charging limit of BESS: 


$$
P_{\mathrm{BESS}, b}^{\max , C}\left(\Delta t_{i}\right)= \begin{cases}0 ; & \text { if } \operatorname{SOC}_{b}\left(\Delta t_{i}\right)=\mathrm{SOC}_{b}^{\max } ; \forall \Delta t_{i} \in T_{\mathrm{BESS}}^{C}, \forall b \in \Omega_{B}, \\ P_{\mathrm{BESS}, b}^{\max , C} & \text { if } \operatorname{SOC}_{b}\left(\Delta t_{i-1}\right)+\frac{\eta_{C} P_{\mathrm{BESS}, b}^{\max , C}}{W_{\mathrm{BESS}, b}^{R}}\left(\Delta t_{i}\right)<\mathrm{SOC}_{b}^{\max } ; \forall \Delta t_{i} \in T_{\mathrm{BESS}}^{C}, \forall b \in \Omega_{B}, \\ \left(\mathrm{SOC}_{b}^{\max }-\operatorname{SOC}_{b}\left(\Delta t_{i}\right)\right) \frac{W_{\mathrm{BESS}, b}^{R} ;}{\Delta t_{i}} & \text { if } \operatorname{SOC}_{b}^{\max }-\operatorname{SOC}_{b}\left(\Delta t_{i-1}\right)<\frac{\eta_{C} P_{\mathrm{BESS}, b}^{\max , C}}{W_{\mathrm{BESS}, b}^{R}}\left(\Delta t_{i}\right) ; \forall \Delta t_{i} \in T_{\mathrm{BESS}}^{C}, \forall b \in \Omega_{B} .\end{cases}
$$

(7) Calculation of maximum discharging limit of BESS:

$$
P_{\mathrm{BESS}, b}^{\max , D}\left(\Delta t_{i}\right)=\left\{\begin{array}{l}
0 \\
P_{\mathrm{BESS}, b}^{\max , D} \\
\left(\mathrm{SOC}_{b}\left(\Delta t_{i}\right)-\mathrm{SOC}_{b}^{\min }\right) \frac{W_{\mathrm{BESS}, b}^{R}}{\Delta t_{i}}
\end{array}\right.
$$

(8) BESS discharging constraint:

$$
\begin{aligned}
& \text { if } \operatorname{SOC}_{b}\left(\Delta t_{i}\right)=\operatorname{SOC}_{b}^{\min } ; \forall \Delta t_{i} \in T_{\mathrm{BESS}}^{S}, \forall b \in \Omega_{B}, \\
& \text { if } \operatorname{SOC}_{b}\left(\Delta t_{i-1}\right)+\frac{P_{\mathrm{BESS}, b}^{\max , D}}{\eta_{D} W_{\mathrm{BESS}, b}^{R}}\left(\Delta t_{i}\right) \geq \mathrm{SOC}_{b}^{\mathrm{min}} ; \forall \Delta t_{i} \in T_{\mathrm{BESS}}^{D}, \forall b \in \Omega_{B}, \\
& \text { if } \operatorname{SOC}_{b}\left(\Delta t_{i-1}\right)-\mathrm{SOC}_{b}^{\min }<\frac{P_{\mathrm{BESS}, b}^{\max , D}}{\eta_{D} W_{\mathrm{BESS}, b}^{R}}\left(\Delta t_{i}\right) ; \forall \Delta t_{i} \in T_{\mathrm{BESS}}^{D}, \forall b \in \Omega_{B} .
\end{aligned}
$$

$$
\operatorname{SOC}_{b}^{\min } \leq \operatorname{SOC}_{b}\left(\Delta t_{i}\right) \leq \operatorname{SOC}_{b}^{\text {max }} ; \quad \forall \Delta t_{i} \in T, \forall b \in \Omega_{B} .
$$

$0 \leq P_{\mathrm{BESS}, b}^{D}\left(\Delta t_{i}\right) \leq P_{\mathrm{BESS}, b}^{\max , D}\left(\Delta t_{i}\right) ; \quad \forall \Delta t_{i} \in T_{\mathrm{BESS}}^{D}, \forall b \in \Omega_{B}$.

(22)
(10) Battery SOC balance constraint during operation:

\section{(9) Battery SOC limit constraint:}

$$
\operatorname{SOC}_{b}\left(\Delta t_{i}\right)=\operatorname{SOC}\left(\Delta t_{i-1}\right)+\left(\frac{\eta_{C, b} P_{\mathrm{BESS}, b}^{C}}{W_{\mathrm{BESS}, b}^{R}}-\frac{P_{\mathrm{BESS}, b}^{D}}{\eta_{D, b} W_{\mathrm{BESS}, b}^{R}}\right) \Delta t_{i} ; \quad \forall \Delta t_{i} \in T_{\mathrm{BESS}}^{C / D}, \forall b \in \Omega_{B} .
$$

(11) Battery SOC balance constraint in standing mode:

$$
\operatorname{SOC}_{b}\left(\Delta t_{i}\right)=\operatorname{SOC}\left(\Delta t_{i-1}\right) ; \quad \forall \Delta t_{i} \in T_{\mathrm{BESS}}^{S}, \forall b \in \Omega_{B} .
$$

2) Reverse power flow constraint:

$$
P_{\text {rev }}\left(\Delta t_{i}\right)=\left\{\begin{array}{l}
0 ; \text { If } \delta_{1}\left(\Delta t_{i}\right)>\delta_{2}\left(\Delta t_{i}\right) ; \\
\Re\left(V_{1}\left(\Delta t_{i}\right) I_{1}^{*}\left(\Delta t_{i}\right)\right) ; \text { else }
\end{array} \forall \Delta t_{i} \in T\right.
$$

\section{Solution Methodology}

The coordinated scheduling of BESSs and MT for maximizing the DPF is a complex nonlinear multiconstraint optimization problem, which is difficult to handle by using conventional analytical-based methods. For this kind of complex combinatorial optimization problems, AI-based nature-inspired metaheuristic or evolutionary methods which may include genetic algorithm (GA) [28], gravitational search algorithm (GSA) [29], African buffalo optimization (ABO) [30], and many more were used to solve it [31]. The ABO algorithm was developed by Odili et al. in 2015 [32]. It is a swarm intelligence-based, nature-inspired, and metaheuristic optimization algorithm motivated by the social and herding behaviour of African buffaloes [33]. It also offers the capability of solving optimization problems suffering from premature convergence. On the other hand, it suffers from the inability to provide global optimum and to tackle complex engineering optimization problems. To overcome some of these limitations, Singh et al. [30] introduced a modified variant of $\mathrm{ABO}$ named $\mathrm{MABO}$ that offers the potential to seek a global or near-global optimum solution for the complex distribution power system optimization problems. Besides, the modifications provide 
well-balanced and well-coordinated exploration and exploitation capabilities. Therefore, in this work, MABO is adopted to solve the considered optimization problem. For further details like necessary steps, proposed modifications in standard variant, mathematical equations, and algorithm parameters of MABO, the readers may refer to [30, 31]. The structure of an individual buffalo is composed of decision variables of the optimization problem. For the proposed problem, the decision variables include charging/discharging dispatch of BESSs and MT unit dispatch. The generalized structure of an individual for the ith system state employed in the present work is shown in Figure 1. The figure shows the structure of dispatches of BESSs and MT units for ith system state.

DMS keeps a record of necessary decisions to be taken for system states through mean price-based adaptive scheduling, as already discussed in Section 2.1. This provides a feasible environment and guidance for $\mathrm{MABO}$ to perform sequential optimization while maximizing the DPF of the utility. In addition, it may alleviate the computational burden of MABO by reducing search space. However, the computational efficiency evaluation is beyond the scope of this work. In this way, MABO sequentially optimizes all system states in order to evaluate the daily profit function of the utility. The flowchart of the proposed methodology, employing MABO as subroutine optimization, is shown in Figure 2.

\section{Case Study}

5.1. Test System. In this section, a $12.67 \mathrm{kV}, 33$-bus standard test distribution system is used to test the validation of the proposed methodology [30]. In this case, the alleged active and reactive load demand is $3.725 \mathrm{MW}$ and $2.50 \mathrm{MVar}$, respectively. With the case distribution system loading, the power losses and the minimum node voltage are $202.66 \mathrm{~kW}$ and 0.9131 p.u. Bus and line data, in this case, can be taken from the reference mentioned above. This system is modified by assuming various existing DERs; the nodes and capacity of distributed energy resources are shown in Table 1.

The reserve on MT unit is taken as $400 \mathrm{~kW}$. The sample day considered for optimal scheduling of BESSs and MT unit is assumed to be composed of 24 states, and every state sustains one hour. For PV and WT, the power generation data on an hourly basis is taken from [25] while ref. [26] may be referred for hourly data of load consumption. The available generation and load data are normalized with respect to the rated capacity of considered DERs and peak load demand, respectively, so as to get hourly generation and load multiplying factors. The dynamic load multiplying factors are assumed to be similar among all buses for a particular system state. These load and renewable generation multiplying factors are further utilized for synthetic data generation by using the modified uncertainty set already discussed in Section 2.2. The profiles of synthetic data generated for load demand and PV and WT power generation are shown in Figure 3.

A dynamic energy pricing scheme is considered to be offered by the transmission network operators. The sale price of energy to customers is also considered dynamic but is assumed 5\% higher for each system state. The day-ahead time-varying price of energy purchase from the grid is taken from [34], as shown in Figure 4. The figure also shows a mean price line and thus defines the subperiod $T_{\mathrm{BESS}}^{D}$ from 09:00 hrs to $23: 00 \mathrm{hrs}$ and $T_{\mathrm{BESS}}^{\mathrm{C}}$ for the remaining hours of the day. Various other parameters considered for simulation purpose are presented in Table 2. The node voltage constraint limits are taken as $\pm 4 \%$. Simulations are executed on a marketable computer parament as Intel Core i7-8600 CPU, $3.80 \mathrm{GHz}$ processor with random access memory of $8 \mathrm{~GB}$. The results obtained for the optimal scheduling of BESSs and MT unit are presented and investigated.

5.2. Simulation Results and Discussions. In this section, the proposed optimization problem is solved by using the MABO presented in Section 4. The pertinent parameters of $\mathrm{MABO}$ are taken as the simulation size is 10 , and the maximum number which can be achieved in these generations is 100. A Backward/forward sweep method is employed to solve the load flow equations. The optimal economic equation obtained under the proposed framework (named as "A") is presented in Table 3. It shows that total payment incurred in purchasing energy from the grid and renewable DGs and in producing energy from the MT unit and BESSs is US\$ $5395.14 \approx 5.40 \mathrm{k}$. The revenue generated by the billing of customers, including the cost of feeder power loss, is US $\$ 7593.42 \approx 7.60 \mathrm{k}$. The profit function of the sample day considered is, therefore, US $\$ 2198.28 \approx 2.20 \mathrm{k}$, which is around $41 \%$. However, the actual profit will be less after deducting the returns against the installation cost of BESSs and MT unit, taxes, etc. It can be acknowledged from the table that almost three-fourths of the payment is incurred for grid power purchase and the rest one-fourth dispersed among local generations.

The energy equation obtained for the proposed optimal scheduling of BESSs and MT is also presented in Table 4. It can be acknowledged from the table that the system deals with 110587.59 units $(\mathrm{kWh})$ of energy out of which about $56 \%$ is imported from the grid and $44 \%$ is matched locally. The contribution of PV and WT units is about $10 \%$ and $20 \%$, respectively, whereas BESSs and MT unit each contributed about $7 \%$. The self-adequacy of the system is of the order of $44 \%$. The table also shows that charging of BESS requires $10364 \mathrm{kWh}$ but delivers $7450 \mathrm{kWh}$ in the system. For the given study, the price of the unit purchase from the local resources is taken cheaper than the dynamic grid price. Therefore, about $75 \%$ of the total payment needed to match grid purchase which is only $56 \%$ of the total energy demand.

To validate the promising profit maximization abilities of the proposed optimization framework, the obtained simulation results are compared with a well-established BESS operation strategy, named as "B". For this purpose, the strategy is applied to this distribution system, and the obtained results are compared with the proposed strategy, as presented in Tables 3 and 5. It has been observed that the daily profit is $3.94 \%$ more, and the losses are $1.50 \%$ less using the proposed strategy. The charging/discharging of BESSs 


\begin{tabular}{c|c|c|c|c|c}
$P_{\mathrm{BESS}, 1}^{\mathrm{C} / \mathrm{D}}\left(\Delta t_{i}\right)$ & $\ldots$ & $P_{\mathrm{BESS}, m}^{\mathrm{C} / \mathrm{D}}\left(\Delta t_{i}\right)$ & $P_{\mathrm{MT}, 1}\left(\Delta t_{i}\right)$ & $\ldots$ & $P_{\mathrm{MT}, r}\left(\Delta t_{i}\right)$ \\
\hline
\end{tabular}

FIGURE 1: Structure of an individual adopted in MABO.

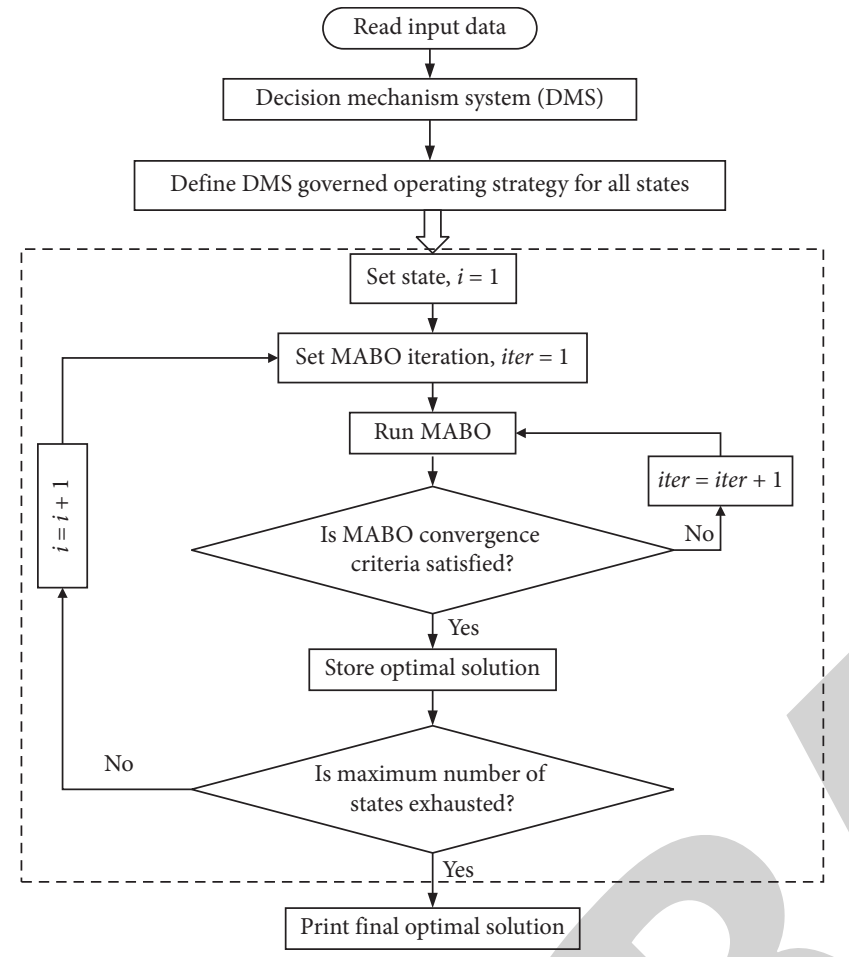

FIGURE 2: Flowchart of the proposed methodology.

TABLE 1: Nodes and capacity of distributed energy resources.

\begin{tabular}{lcccc}
\hline DER nodes & & 17 & 25 & 30 \\
\hline \multirow{5}{*}{ DER capacities } & PV (kWp) & 2100 & - & - \\
& WT (kWp) & - & - & 1900 \\
& MT (kW) & - & 1100 & - \\
& BESS (kWh) & 3000 & 3000 & 3000 \\
\hline
\end{tabular}

using these two strategies is compared in Figure 5. It can be acknowledged from the figure that the charging of BESSs is almost the same using both the strategies; however, the discharging is quite different. The strategy discharges BESSs immediately after full charging that shifts standby mode of BESSs to peak hours. Thus, this strategy is unable to exploit peak shaving and associated technical benefits. But the proposed strategy suitably manages standby periods of BESSs in such a way that they essentially discharge during on-peak hours. This shows that BESSs can be managed in a better way using the proposed MPAS.

After validation of the proposed optimization framework, its promising features have been investigated further by presenting the analysis of each state. The optimal solution provides profit function for each state, as presented in Figure 6. It can be observed that the profit function becomes negative during certain off-peak hours. It happened because additional energy is being drawn from the grid or DGs

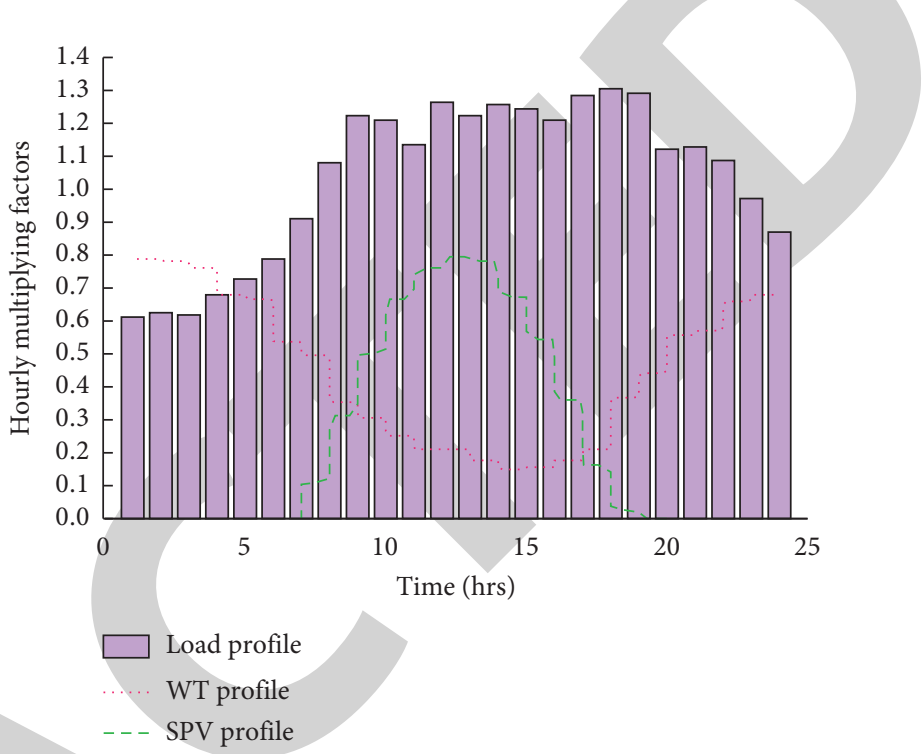

Figure 3: Synthetic data generated for PV and WT power generation and load demand.

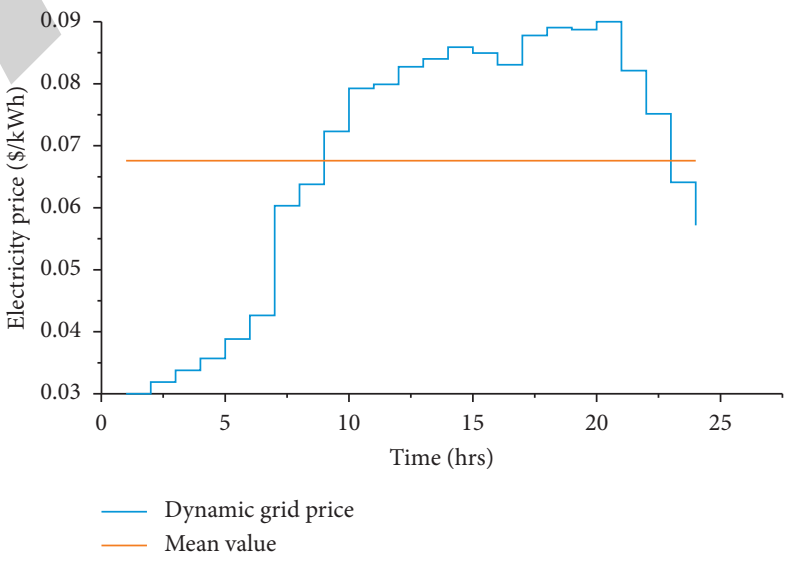

Figure 4: Day-ahead dynamic grid price over one day.

against the charging of BESSs and thus increases payments and supersedes revenue owing to lesser off-peak demand. The DPF, however, is found to be US\$ 2198.28 with a positive sign, as also shown in Table 3 . A dip in the profit function is observed during subperiod $T_{B E S S}^{D}$ which is on account of dip in load demand as well as dynamic price and standby mode of all BESSs. Otherwise, it maintains moderately higher positive values during most of the peak pricing hours, i.e., 13:00 hrs and 17:00 hrs to 19:00 hrs.

The optimal scheduling and dispatches of BESSs and MT unit obtained using the proposed method are presented in Figure 7. It can be observed that all BESSs exclusively charge and discharge during subperiods $T_{\mathrm{BESS}}^{C}$ and $T_{\mathrm{BESS}}^{D}$, respectively, and these two subperiods are separated by standby 
TABLE 2: Simulation parameters.

\begin{tabular}{lccc}
\hline Simulation parameter & Value & Simulation parameter & Value \\
\hline$H$ & $85 \%$ & $M_{\mathrm{BESS}}$ & $0.003 \$ / \mathrm{kWh}$ \\
$P_{\mathrm{BESS}, b}^{\max , C}, P_{\mathrm{BESS}, b}^{\max , D}$ & $500 \mathrm{~kW}, 500 \mathrm{~kW}$ & $M_{M T}$ & $0.011 \$ / \mathrm{kWh}$ \\
$\mathrm{SOC}_{b}^{\min }, \mathrm{SOC}_{b}^{\max }$ & $0.1,1.0$ & $E_{P V}$ & $0.026 \$ / \mathrm{kWh}$ \\
$\mathrm{SOC}_{I}$ & 0.1 & $E_{W T}$ & $0.029 \$ / \mathrm{kWh}$ \\
$E_{\mathrm{BESS}}^{F}$ & $0.065 \$ / \mathrm{kWh}$ & $E_{M T}$ & $0.035 \$ / \mathrm{kWh}$ \\
\hline
\end{tabular}

TABle 3: Energy equation over the scheduling period of 24 hours (MWh and \%).

\begin{tabular}{|c|c|c|c|c|c|c|c|c|c|c|}
\hline \multirow{2}{*}{ Method } & \multicolumn{3}{|c|}{ Purchased } & \multicolumn{2}{|c|}{ Supplied } & \multirow{2}{*}{ Total (MWh) } & \multicolumn{3}{|c|}{ Consumed } & \multirow{2}{*}{ Total (MWh) } \\
\hline & Grid & PV & WT & MT & BESS & & Billing & Loss & BESS & \\
\hline Proposed & $62.15 / 56.16$ & $\begin{array}{l}11.35 / \\
10.26\end{array}$ & $\begin{array}{c}21.69 / \\
19.63\end{array}$ & $\begin{array}{l}8.00 / \\
7.24\end{array}$ & $\begin{array}{c}7.45 / \\
6.73\end{array}$ & 110.61 & $\begin{array}{c}96.68 / \\
87.45\end{array}$ & $\begin{array}{l}3.58 / \\
3.22\end{array}$ & $\begin{array}{c}10.36 / \\
9.38\end{array}$ & 110.59 \\
\hline Analytical & $\begin{array}{c}62.32 / \\
56.28 \\
\end{array}$ & $\begin{array}{c}11.35 / \\
10.25 \\
\end{array}$ & $\begin{array}{c}21.69 / \\
19.58 \\
\end{array}$ & $\begin{array}{l}8.00 / \\
7.22 \\
\end{array}$ & $\begin{array}{l}7.38 / \\
6.65 \\
\end{array}$ & 110.72 & $\begin{array}{r}96.68 / \\
87.29 \\
\end{array}$ & $\begin{array}{l}3.60 / \\
3.25 \\
\end{array}$ & $\begin{array}{c}10.45 / \\
9.43\end{array}$ & 110.73 \\
\hline
\end{tabular}

TABLE 4: Comparison of energy equation over the scheduling period of 24 hours (MWh and \%).

\begin{tabular}{|c|c|c|c|c|c|c|c|c|c|c|}
\hline \multirow{2}{*}{ Method } & \multicolumn{3}{|c|}{ Purchased } & \multicolumn{2}{|c|}{ Supplied } & \multirow{2}{*}{$\begin{array}{c}\text { Total } \\
\text { (MWh) }\end{array}$} & \multicolumn{3}{|c|}{ Consumed } & \multirow{2}{*}{$\begin{array}{l}\text { Total } \\
\text { (MWh) }\end{array}$} \\
\hline & Grid & SPV & WT & MT & BESS & & Billing & Loss & BESS & \\
\hline Analytical method & $62.33 /$ & $11.35 /$ & $21.69 /$ & $8.00 /$ & 7.361 & & $96.68 /$ & $3.60 /$ & $10.45 /$ & \\
\hline [10] & 56.28 & 10.25 & 19.58 & 7.22 & 6.65 & & 87.30 & 3.25 & 9.43 & 110.13 \\
\hline Proposed method & $\begin{array}{c}62.10 / \\
56.15\end{array}$ & $\begin{array}{c}11.35 \\
10.26\end{array}$ & $\begin{array}{c}21.69 / \\
19.61\end{array}$ & $\begin{array}{l}8.00 / \\
7.23\end{array}$ & $\begin{array}{c}7.45 / \\
6.74\end{array}$ & 110.59 & $\begin{array}{c}96.68 / \\
87.42\end{array}$ & $\begin{array}{c}3.55 / \\
3.20\end{array}$ & $\begin{array}{c}10.36 / \\
9.37\end{array}$ & 110.59 \\
\hline
\end{tabular}

TABLE 5: Economic equation over the scheduling period of 24 hours (in US $\$ \times 10^{3}$ and \%).

\begin{tabular}{|c|c|c|c|c|c|c|c|c|c|c|c|}
\hline \multirow[b]{2}{*}{ Method } & \multirow[b]{2}{*}{ Grid } & \multirow[b]{2}{*}{ PV } & \multirow{2}{*}{\multicolumn{2}{|c|}{ Cost }} & \multirow[b]{2}{*}{$\begin{array}{c}\text { MT } \\
(\mathrm{OM})\end{array}$} & \multirow[b]{2}{*}{$\begin{array}{l}\text { BESS } \\
(\mathrm{OM})\end{array}$} & \multirow[b]{2}{*}{ Total } & \multicolumn{2}{|c|}{ Revenue } & \multirow[b]{2}{*}{ Total } & \multirow[b]{2}{*}{ Profit } \\
\hline & & & & & & & & $\begin{array}{c}\text { Cost of } \\
\text { consumption }\end{array}$ & $\begin{array}{l}\text { Cost of } \\
\text { losses }\end{array}$ & & \\
\hline Proposed & $4.08 / 75.2$ & $\begin{array}{c}0.32 / \\
5.90\end{array}$ & $\begin{array}{l}0.63 / \\
11.76\end{array}$ & $\begin{array}{l}0.27 / \\
4.97\end{array}$ & $0.10 / 1.79$ & $0.03 / 0.49$ & 5.41 & $7.34 / 96.71$ & $0.25 / 3.32$ & 7.61 & $\begin{array}{l}2.20 / \\
40.78\end{array}$ \\
\hline Analytical & $\begin{array}{l}4.15 / \\
75.63\end{array}$ & $\begin{array}{c}0.32 / \\
5.78\end{array}$ & $\begin{array}{l}0.63 / \\
11.45\end{array}$ & $\begin{array}{c}0.27 / \\
4.86\end{array}$ & $0.10 / 1.74$ & $0.03 / 0.48$ & 5.50 & $7.34 / 96.58$ & $0.26 / 3.41$ & 7.61 & $\begin{array}{l}2.11 / \\
38.44\end{array}$ \\
\hline
\end{tabular}

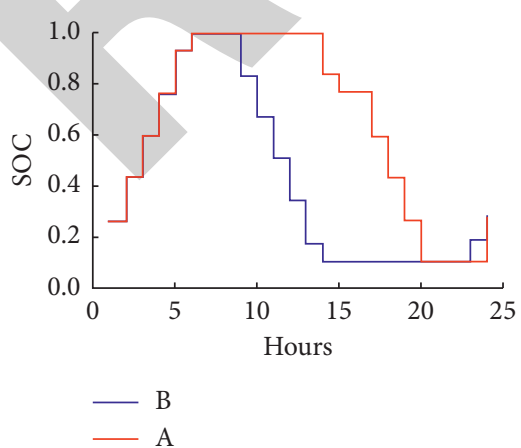

(a)

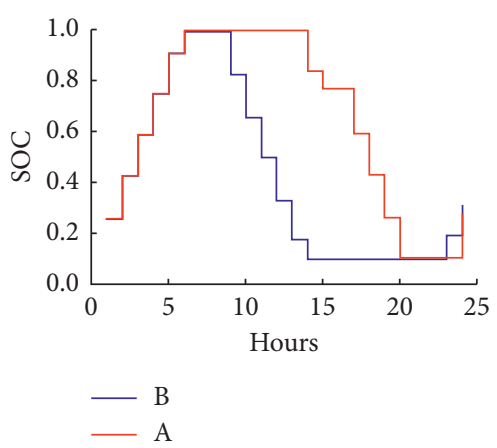

(b)

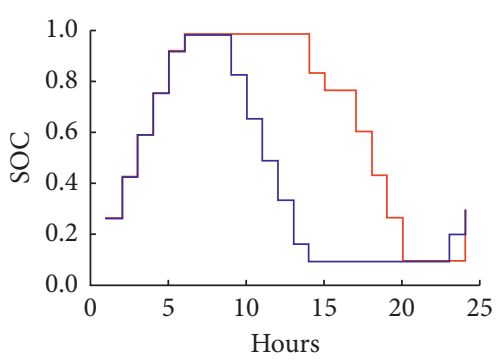

$-\mathrm{A}$

FIGURE 5: Optimal dispatch/SOC of BESSs obtained by the proposed approach (A) and existing approach (B). (a) BESS at node 9. (b) BESS at node 24. (c) BESS at node 32. 


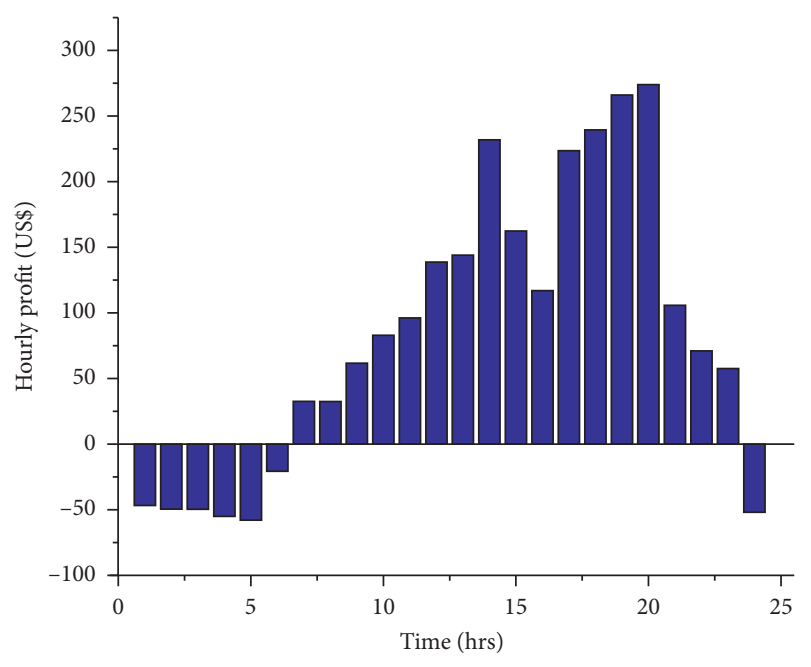

FIGURE 6: Values of hourly profit function obtained under the proposed optimization framework.

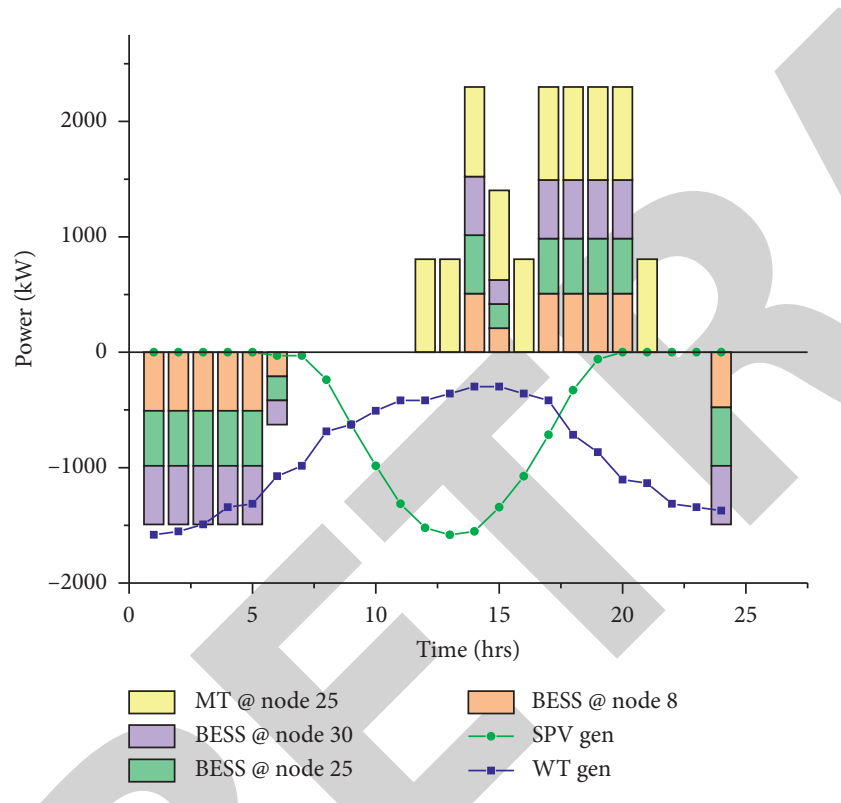

FIGURE 7: Optimal scheduling of all BESSs and MT.

period. As shown in Figure 5, the system takes full advantage of all BESSs as their SOC status reached the same level in one round trip. Total energy dispatch from these DERs is found to be about $32 \%$ of the energy demand that prevails during on-peak hours, i.e., $12: 00 \mathrm{hrs}$ to $21: 00 \mathrm{hrs}$. It can be observed that BESSs charge mostly from WT and discharge during peak pricing hours where solar power is not much available. Thus, BESSs neutralize the intermittency of wind power and deliver the same during acute operating conditions of the system, besides gaining charge differential benefits. The power generated from PV and WT is deliberately shown with a negative sign merely to have a better understanding.

The modification in load profile while employing optimal dispatches from BESSs and MT is shown in Figure 8.

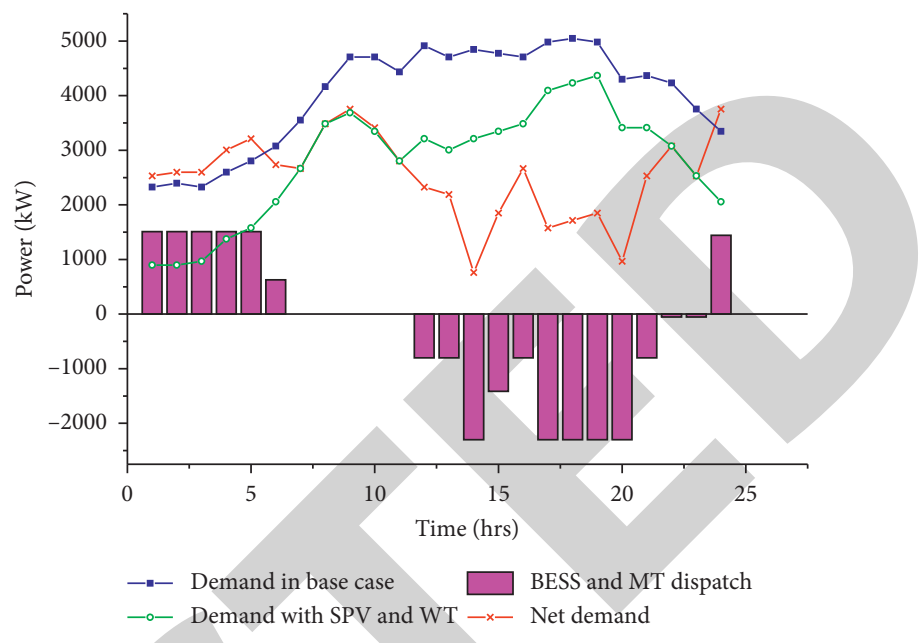

Figure 8: Load profile of the system under different scenarios.

Considering optimal dispatches from BESSs and MT unit, the load profile and the net load profile under different scenarios can be acknowledged from the figure. It can be observed that the fluctuations in load profile increase using renewables and that further worsen using BESSs and MT unit. However, mean and peak demand are found to be reduced by $9.87 \%$ and $14.71 \%$ whereas the valley demand is reduced by $11.99 \%$ using optimal scheduling of BESSs and MT. Moreover, the peak demand is shifted from 19:00 hrs to 09 : $00 \mathrm{hrs}$, whereas the new valley demand occurred at 14: $00 \mathrm{hrs}$ instead of $01: 00 \mathrm{hrs}$. Another useful finding is observed while comparing the load profiles. The load deviation index (LDI) is evaluated for these profiles. The index is a standard deviation of the demands constituting the load profile. The smaller value of this index is desirable. The LDI of load demand is found to be $924.38 \mathrm{~kW}$ which is deteriorated to $1031.07 \mathrm{~kW}$ by the integration of PV and WT units. However, it is improved to $765.45 \mathrm{~kW}$ using optimal scheduling of BESSs and MT unit though the scheduling was determined on an economic basis. Thus, pure economic optimal scheduling of BESSs and MT causes moderate peak demand shaving and valley deepening, partial rebound effect, and enhanced LDI, but with somewhat fluctuating load demand. The fluctuations in load demand attributed to maximizing price differential benefits using proposed scheduling during peak pricing hours. The net demand depicts no reverse power flow and thus ensures better coordination of existing energy resources.

\section{Conclusion}

This paper addresses the optimal utilization of existing dispatchable and nondispatchable energy resources by coordinating utility-owned BESSs and MT unit to maximize daily profit function (DPF) while accounting for uncertainty in nondispatchable resources and load demand. The proposed methodology introduces mean price-based adaptive scheduling (MPAS) being embedded within a decision mechanism system (DMS). The proposed algorithm and fictitious charges for BESS operation in dynamic pricing 
provide necessary information to DMS. DMS processes the information as a priori for possible maximization of profit function and thus guides MABO for the concurrent state. The application results obtained on a standard test bench shows that MPAS provides economic system operation and better exploitation of available energy resources and improves LDI. The comparison of the proposed strategy with the existing one shows an enhanced profit of the utility and better utilization and management of existing energy resources besides power loss reduction. However, peak hours may face some fluctuations in load demand on account of gaining price differential benefits. This highlights a possible limitation that may arise while employing pure economic optimal scheduling of BESSs and MT. Either multiobjective optimization or ancillary services may overcome this limitation. Demand response is ignored in the present work but can play a vital role in deciding load profile and load fluctuations. The present work may be extended to explore these concerns. Further, the conclusions can be made that the proposed flexible and adaptive scheduling strategy will be promising to operate BESSs in future energy networks.

\section{Data Availability}

The experiment data used to support the findings of this study are included within the article.

\section{Conflicts of Interest}

The authors declare that they have no conflicts of interest.

\section{Acknowledgments}

This study was funded by Economic and Electrical Research Institute of Shanxi Electrical Power Company of SGCC (Research on power grid planning method and development path in remote areas under the background of modern energy system).

\section{References}

[1] C. Guo, F. Luo, Z. Cai et al., "Integrated planning of internet data centers and battery energy storage systems in smart grids," Applied Energy, vol. 281, 2021.

[2] F. Tooryan, H. HassanzadehFard, E. R. Collins, S. Jin, and B. Ramezani, "Smart integration of renewable energy resources, electrical, and thermal energy storage in microgrid applications," Energy, vol. 212, p. 118716, 2020.

[3] M. Farrokhifar, S. Grillo, and E. Tironi, "Loss minimization in medium voltage distribution grids by optimal management of energy storage devices," in Proceedings of the 2013 IEEE Grenoble Conference, pp. 1-5, Grenoble, France, November 2013.

[4] M. Farrokhifar, S. Grillo, and E. Tironi, "Optimal placement of energy storage devices for loss reduction in distribution networks," in Proceedings of the IEEE PES-ISGT Europe, pp. 1-5, Copenhagen, Denmark, October 2013.

[5] T. B. Reddy, Linden's Handbook of Batteries, Vol. 4, McGraw-Hill, New York, NY, USA, 2011.

[6] L. A. Hannah and D. B. Dunson, "Approximate dynamic programming for storage problems," in Proceedings of the 28th
International Conference on Machine Learning (ICML-11), pp. 337-344, Omnipress, Bellevue, WA, USA, June 2011.

[7] S. Grillo, A. Pievatolo, and E. Tironi, "Optimal storage scheduling using Markov decision processes," IEEE Transactions on Sustainable Energy, vol. 7, no. 2, pp. 755-764, 2016.

[8] F. D. Galiana, F. Bouffard, J. M. Arroyo, and J. F. Restrepo, "Scheduling and pricing of coupled energy and primary, secondary, and tertiary reserves," Proceedings of IEEE, vol. 93, no. 11, pp. 1970-1983, 2005.

[9] B. Zeng, Y. Liu, F. Xu, Y. Liu, X. Sun, and X. Ye, "Optimal demand response resource exploitation for efficient accommodation of renewable energy sources in multi-energy systems considering correlated uncertainties," Journal of Cleaner Production, vol. 288, p. 125666, 2020.

[10] N. Gast, D.-C. Tomozei, and J.-Y. Le Boudec, "Optimal generation and storage scheduling in the presence of renewable forecast uncertainties," IEEE Transactions on Smart Grid, vol. 5, no. 3, pp. 1328-1339, 2014.

[11] A. I. Bejan, R. J. Gibbens, and F. P. Kelly, "Statistical aspects of storage systems modelling in energy networks," in Proceedings of the 46th Annual Conference on Information Sciences and Systems (CISS), Princeton, NJ, USA, March 2012.

[12] Y. Jiang, L. Kang, and Y. Liu, "Optimal configuration of battery energy storage system with multiple types of batteries based on supply-demand characteristics," Energy, vol. 206, p. $118093,2020$.

[13] J. Xiao, Z. Zhang, L. Bai, and H. Liang, "Determination of the optimal installation site and capacity of battery energy storage system in distribution network integrated with distributed generation," IET Generation, Transmission \& Distribution, vol. 10, no. 3, pp. 601-607, 2016.

[14] J. Lei and Q. Gong, "Operating strategy and optimal allocation of large-scale VRB energy storage system in active distribution networks for solar/wind power applications," IET Generation, Transmission \& Distribution, vol. 11, no. 9, pp. 2403-2411, 2017.

[15] J. Chen, Y. Liu, and G. Bao, "Optimal operating strategy for distribution networks with PV and BESS considering flexible energy storage," in Proceedings of the 2016 IEEE Power and Energy Society General Meeting (PESGM), Boston, MA, USA, June 2016.

[16] B. Zeng, J. Feng, N. Liu, and Y. Liu, “Co-optimized parking lot placement and incentive design for promoting PEV integration considering decision-dependent uncertainties," IEEE Transactions on Industrial Informatics, vol. 17, no. 3, pp. 1863-1872, 2021.

[17] J. Qin, R. Sevlian, D. Varodayan, and R. Rajagopal, "Optimal electric energy storage operation," in Proceedings of the 2012 IEEE Power and Energy Society General Meeting, pp. 1-6, San Diego, CA, USA, July 2012.

[18] S. Harsh, "Wake management based life enhancement of battery energy storage system for hybrid wind farms," Renewable and Sustainable Energy Reviews, vol. 130, p. 109912, 2020.

[19] M. R. Jannesar, A. Sedighi, M. Savaghebi, and J. M. Guerrero, "Optimal placement, sizing, and daily charge/discharge of battery energy storage in low voltage distribution network with high photovoltaic penetration," Applied Energy, vol. 226, pp. 957-966, 2018.

[20] I. B. Sperstad and M. Korpås, "Energy storage scheduling in distribution systems considering wind and photovoltaic generation uncertainties," Energies, vol. 12, no. 7, 2019. 
[21] J. Koskela, A. Mutanen, and P. Järventausta, "Using load forecasting to control domestic battery energy storage systems," Energies, vol. 3946, pp. 13-15, 2020.

[22] J. Qiu, J. Zhao, H. Yang, D. Wang, and Z. Y. Dong, "Planning of solar photovoltaics, battery energy storage system and gas micro turbine for coupled micro energy grids," Applied Energy, vol. 219, pp. 361-369, 2018.

[23] B. Zeng, H. Dong, R. Sioshansi, F. Xu, and M. Zeng, "Bilevel robust optimization of electric vehicle charging stations with distributed energy resources," IEEE Transactions on Industry Applications, vol. 56, no. 5, pp. 5836-5847, 2020.

[24] B. Zeng, J. Zhang, X. Yang, J. Wang, J. Dong, and Y. Zhang, "Integrated planning for transition to low-carbon distribution system with renewable energy generation and demand response," IEEE Transactions on Power Systems, vol. 29, no. 3, pp. 1153-1165, 2014.

[25] National Renewable Energy Laboratory (NREL), 2020, https://www.nrel.gov.

[26] Power System Operation Corporation Limited (A GoI Enterprise), 2020, https://posoco.in/.

[27] B. L. Gorissen, İ. Yanıkoğlu, D. den Hertog, and D. den Hertog, "A practical guide to robust optimization," Omega, vol. 53, pp. 124-137, 2015.

[28] B. Zeng, Z. Zhu, H. Xu, and H. Dong, "Optimal public parking lot allocation and management for efficient PEV accommodation in distribution systems," IEEE Transactions on Industry Applications, vol. 56, no. 5, pp. 5984-5994, 2020.

[29] S. Singh and M. Fozdar, "Double-sided bidding strategy for power suppliers and large buyers with amalgamation of wind and solar based generation in a modern energy market," IET Generation, Transmission \& Distribution, vol. 14, no. 6, pp. 1031-1041, 2019.

[30] P. Singh, N. K. Meena, A. Slowik, and S. K. Bishnoi, "Modified African buffalo optimization for strategic integration of battery energy storage in distribution networks," IEEE Access, vol. 8, pp. 14289-14301, 2020.

[31] M. E. Baran and F. F. Wu, "Network reconfiguration in distribution systems for loss reduction and load balancing," IEEE Transactions on Power Delivery, vol. 4, no. 2, pp. 14011407, 1989.

[32] J. B. Odili, M. N. M. Kahar, and S. Anwar, "African buffalo optimization: a swarm-intelligence technique," Procedia Computer Science, vol. 76, pp. 443-448, 2015.

[33] D. S. Wilson, "Altruism and organism: disentangling the themes of multilevel selection theory," The American Naturalist, vol. 150, no. 1, pp. 122-S134, 1997.

[34] Indian Energy Exchange (IEX), 2020, https://www.iexindia. $\mathrm{com} /$. 